\title{
PROCESS AND SYSTEMS Beyond financial efficiency to support environmental sustainability in economic evaluations
}

\author{
Authors: Laure de Preux ${ }^{A}$ and Dheeya Rizmie ${ }^{B}$
}

\begin{abstract}
The healthcare sector is one of the largest polluters in the UK, accounting for $\mathbf{2 5 \%}$ of total emissions of carbon dioxide of the public sector. Ironically, it is the healthcare sector itself that is primarily affected by any deterioration in the environment affecting individuals' health and their demand for healthcare. Therefore, the healthcare sector is a direct beneficiary of its own steps towards sustainability and is more and more viewed as the one who should lead the change. In this article, we first review the concepts of financial and environmental sustainability. Second, we discuss the existing evidence of sustainable changes within this sector. Third, we propose a simple adaptation of the classic cost-effectiveness analysis to incorporate carbon footprinting to account for these external costs. We illustrate our method using the case of in-centre versus home haemodialysis. We conclude that home dialysis is always a preferable alternative to in-centre treatment based on a cost-effectiveness analysis. Finally, we discuss the limitations of our approach and the future research agenda.
\end{abstract}

KEYWORDS: Economic evaluation, carbon footprint, environmenta sustainability, ICER

\section{Introduction}

The concept of 'sustainability' is wide-ranging, with its core principle being the ability to 'meet current needs without compromising the ability to meet future needs.' Within healthcare, it is often a term used to reference the financial sustainability of a health system. The financial sustainability of the healthcare sector is a well-accepted requirement and often a major source of concern. With the NHS in the UK under persistent financial stress, efficiency improvements are regularly implemented. ${ }^{2}$ In order to promote efficiency, decision making in the allocation of resources has relied on economic evaluation. The objective is to produce the greatest return in health outcomes with limited financial resources. The standard approach of an economic evaluation compares the financial costs and effectiveness between alternative interventions, services or programmes. Priority is given to services that are determined to

Authors: ${ }^{A}$ assistant professor, Imperial College Business School, Imperial College London, London, UK; ${ }^{\mathrm{B}} \mathrm{PhD}$ candidate, Imperial College Business School, Imperial College London, London, UK be value for money, without any consideration for their long-term environmental impacts. ${ }^{3}$

However, alongside the rise of a broader sustainable policy agenda, the term 'sustainability' is shifting to represent the reconciliation of the financial, societal and environmental pressures that influence current resource use without significantly depleting what is available for the future generations. Therefore, interests in the environmental sustainability of the healthcare system are beginning to emerge. In 2008, the Sustainable Development Unit (SDU) was established by the NHS to more systematically assess the health sector's environmental impact and encourage focus on the wider sustainability triad of environmental, societal and financial needs.

The increasing interest in the environmental sustainability of the healthcare sector can be explained by two key elements. First, the healthcare sector is a large polluter. The Climate Change Act 2008 sets for the UK an $80 \%$ reduction in net emissions by 2050 , based on the 1990 baseline. At the time the SDU was formed, greenhouse gases ( $\mathrm{GHG}$ ) emissions associated to the NHS represented $25 \%$ of public sector emissions in the UK, ${ }^{4}$ making the NHS a necessary source of reduction. Since 2007 , there has been an $11 \%$ reduction in carbon emissions and the carbon footprint of the NHS was reported to be $22.8 \mathrm{MtCO}_{2}$ eq in 2015 , with procurement accounting for $57 \%$ of the total emissions. ${ }^{5}$ Further reductions are necessary for the healthcare sector to meet the 2050 target.

Second, the NHS is also responsible for emitting numerous pollutants and these have direct and indirect impacts on individuals. Different pollutants have been found to be associated with a range of effects on human health, including increased mortality, hospitalisations and emergency admissions, exacerbations of chronic respiratory conditions and decreased lung function. ${ }^{6}$ Pollution is currently the largest environmental cause of global mortality and morbidity, with an estimated 9 million premature deaths in $2015 .{ }^{6}$ As such, healthcare activity becomes a source of illness, contradicting the NHS's primary objective to improve individuals' health and wellbeing. ${ }^{7}$

The environmental sustainability of the healthcare sector is essential for the overall sustainability of healthcare delivery in the future. Current decision making is mostly driven by economic evaluations, which focus primarily on efficient allocation of resources without accounting for the environmental impact of alternative interventions. The underlying rationale of an economic evaluation is to maximise the allocation of resources at the smallest financial cost, in order to ensure the optimal utilisation 
of a limited resource. Traditional cost-effectiveness, cost-benefit, and cost-utility analyses differ in terms of health measurement, but their cost dimension only captures the use of resources within the NHS. While broader costs can be included if a different payer perspective is taken, such as the costs incurred by the patient, the inclusion of any particular cost relies on its market price. As pollution currently still does not have an approved market value, it is simply ignored from analysis. Economists call this an externality. Externalities appear when the healthcare intervention has an impact beyond the healthcare provider and the patient involved in the treatment, and this impact is not accounted for in the transaction.

In this article, we illustrate how the essence of an economic evaluation can be modified to account for carbon emissions of two different treatments. In addition to the financial cost of the interventions, our analysis includes the monetary value of carbon dioxide equivalent $\left(\mathrm{CO}_{2} \mathrm{eq}\right)$ emissions. The method and decision process remain the same but the environmental damages of the two alternatives are now taken into account.

\section{Current approaches to environmental sustainability}

In theory, it is possible for environmentally friendly changes to take place at all levels of the healthcare delivery chain. Naylor and Appleby ${ }^{8}$ explore all the different opportunities for changes across the NHS organisation and argue that integration of sustainability should happen at three main levels: where care is delivered, what care is delivered, and how care is delivered. However, all of these changes can only be successful if accompanied by changes in behaviours, attitudes and cultures across the healthcare organisation. McGain and Naylor ${ }^{9}$ systematically review the literature on environmental sustainability in hospitals. The UK, in particular, appears to have a systematic approach to understanding hospitals' environmental impacts. ${ }^{9}$ Some hospitals have already implemented successful changes, such as the University College London Hospital NHS Foundation Trust which achieved a $14 \%$ carbon reduction by the end of fiscal year $2011 .{ }^{8}$ Voluntary initiatives are also a driver of change but are unlikely to be sufficient in the long-term. ${ }^{10}$ Patrick et $a l^{11}$ review the key barriers to implement changes using case studies and key stakeholder interviews. Unsurprisingly, obstacles are observed at all levels of the healthcare delivery process: individual, organisational and community levels. Others have also highlighted the complexity of contextual factors in the implementation of more environmentally friendly changes. ${ }^{12}$

Beyond these managerial and organisational challenges, there remains a lack of clarity on how to translate sustainable development and available evidence into practice while maintaining and improving efficient delivery of public services. ${ }^{9}$ Guidance has started to emerge on how to account for GHG emissions, as well as waste, ${ }^{13}$ but the available metrics remain limited. ${ }^{14}$ Furthermore, it is not clear how to embed them into existing practice and integrate them into the decision-making process. ${ }^{15}$ The development of measures that "can be used by health and social care organisations, regulators, policy-makers and members of the public to evaluate the environmental impact of different interventions, pathways, technologies and approaches will be critically important'. ${ }^{8}$ In the next section, we propose a simple adjustment of an efficiency metric that accounts for the carbon footprint of a treatment, which allows us to account for GHG emissions in the decision-making process.

\section{Toward an environmentally friendly economic assessment of healthcare}

We propose a simple adjustment to an efficiency assessment that takes into account some environmental damages in the assessment of a healthcare technology. We compare in-centre versus home haemodialysis accounting for their carbon emissions. Haemodialysis (HD) involves diverting blood into an external machine containing a semi-permeable membrane to be filtered before being returned to the body in patients with chronic kidney failure. HD can be undertaken in three main settings: in-centre (usually hospitals), satellite units, and patients' homes. We do not consider $\mathrm{HD}$ in a satellite unit as the cost can vary considerably between different settings. ${ }^{16}$ Home haemodialysis (HHD) and in-centre haemodialysis (ICHD) are associated with the same potential complications, ${ }^{17}$ which therefore allows us to focus mainly on the cost component of the analysis. Conventional HD regimes are administered for 4 hours three times per week. ${ }^{17}$ However, it is possible for a patient to undergo regimes of different frequencies and durations. We compare the first and second years of $\mathrm{HD}$ treatment only, assuming that the subsequent years are not different from the second year, once the setting-up costs have been taken into account.

On one hand, a National Institute for Clinical Excellence (NICE) assessment published in 2002 reviewed the evidence on threetimes weekly dialysis to conclude that HHD is cheaper than ICHD. ${ }^{17}$ On the other hand, $\mathrm{CO}_{2}$ eq emissions were reported to be higher for ICHD, yet the treatment was administered at a different dose in this study. ${ }^{18}$ We combine existing evidence by considering both the financial costs as well as some environmental costs, which has not been previously conducted in the literature, and reconcile the results by comparing the estimates for different doses.

We extract the financial costs from a cost model built by Komenda et al. ${ }^{19}$ The model provides a sum of all component costs for the first year of HD and maintenance in subsequent years. The model considers direct and indirect costs related to the treatment. One-time costs such as dialysis training costs, medications, professional reimbursement and emergency dialysis sessions are accounted for. Standard medical and nonmedical costs have also been included (eg transportation to and from dialysis facilities).

The environmental damage of HD treatments is captured by their carbon footprint. There are several GHG with potential to cause environmental damage. As such, carbon dioxide $\left(\mathrm{CO}_{2}\right)$ is most commonly used as a reference gas, with other gaseous emissions being expressed in units of $\mathrm{CO}_{2}$ equivalents. The $\mathrm{CO}_{2} \mathrm{eq}$ are derived from a carbon footprint assessment conducted by Connor et $a l^{18}$ for the maintenance of HD. The carbon footprint estimates represent the direct and indirect GHG emissions derived from energy use, patient and staff travel and procurement attributable to the provision of maintenance HD by ICHD and HHD. Procurement emissions relate to the acquisition of consumable products required to provide treatment, from the procurement of medical equipment, paper and office supplies, laundry services, construction, water, sanitation products, including chemicals for the external decontamination of HD machines, to the collection, treatment and disposal of waste. Naturally, the frequency and duration of treatments influence the emissions associated with its delivery. ${ }^{18}$

Two main deviations between the financial and environmental estimates should be noted. Firstly, environmental costs in Connor 
et $a l^{18}$ do not include components associated with the start-up and initiation of $\mathrm{HD}$ in the first year of treatment. We apply a $25 \%$ uplift to $\mathrm{CO}_{2}$ eq estimates to conservatively account for this variation, yet the conclusion remains the same even without this adjustment. Second, the GHG emissions attributable to the production of dialysis machines are not accounted for in the carbon estimates, although this omission is likely to be similar in the ICHD and HHD cases and therefore would cancel out.

We follow the UK government's carbon valuation guidance ${ }^{20}$ to estimate the cost of GHG emissions. As all our estimates are set in the year 2010, we apply the non-traded price of carbon (nTPC) to GHG estimates for each HD modality. The nTPC reflects the marginal abatement cost required to meet emission reduction targets and includes the social cost of carbon, which aims to encompass the lifetime damage costs associated with GHG emissions. We use the central value of nTPC in 2010, priced at $£ 52$ per ton $\mathrm{CO}_{2}$ eq. $^{20}$

Quality-adjusted life years (QALYS) are used as the measure of effectiveness of dialysis. We obtain the QALYS gained for 2 years of dialysis from published studies. ${ }^{21,22}$

Table 1 reports financial and $\mathrm{CO}_{2}$ eq costs of ICHD on a 4-hour three times per week regime (first two columns), HHD for the same regime (columns 3 and 4 ), and home nocturnal HD (HNHD) on a 7-hour six times per week regime (columns 5 and 6 ) as this is a more conventional regime when given the opportunity to have $\mathrm{HD}$ at home. It frees patient's time and is associated with higher QALYS.

Looking at the cost components first, ICHD is always more expensive than $\mathrm{HHD}$, even when we account for the $\mathrm{CO}_{2} \mathrm{eq}$, with or without an uplift in the first year. In contrast, HNHD is always more expensive than ICHD and HHD. The same conclusion is reached whether the price of carbon is included or not as, in relative terms, the carbon value is small compared to the overall financial costs. Therefore, purely based on financial and carbon costs, it appears that HHD is a cheaper option, at a conventional frequency of 4 hours three times per week. This result aligns with the conclusions made by NICE. ${ }^{17}$
Once we take into account the difference in health benefits between the different regimes, and calculate the incremental cost-effectiveness ratio (ICER), using the ICHD as our baseline option, the home options are the most cost-effective alternatives. HHD's ICER of $-£ 1,016.46$ in the first year, and - £11,061.37 in the second year suggest that each additional QALY obtained, in this case comparing HHD to ICHD, achieves a saving within the acceptability range of $£ 20,000$ to $£ 30,000$ per QALY used by NICE. ${ }^{3}$ Therefore, HHD of a 4 -hours three times a week regime is considered a superior alternative to ICHD. It achieves higher health benefits per financial and carbon cost.

While HNHD appears to be a costly option, it is nevertheless a more effective solution for the patient. The HNHD's ICER is $£ 14,007.92$ in the first year, and $£ 15,308.78$ in the second year, suggesting that larger health gains can be achieved for a cost well below the $€ 30,000$ per QALY acceptability threshold, even when accounting for carbon costs. ${ }^{23}$

In this simple example, the inclusion of environmental costs supports the financial conclusion based on cost-effectiveness, but the analysis of a different intervention could reach an opposite conclusion should the environmental costs be large enough to outweigh the financial costs.

\section{Discussion}

The analysis of ICHD versus at HHD and HNHD illustrates how to account for carbon footprint in the case of a treatment. In proportion to the financial costs, the carbon cost was relatively small and did not lead to a different conclusion solely based on financial cost. Once the health benefits are taken into account, HNHD appears to be a cost-effective alternative despite its large cost. Currently, only $4.2 \%$ of all dialysis patients in the UK are receiving home $\mathrm{HD},{ }^{24}$ but our results support a shift to home $\mathrm{HD}$ whenever it can be an option for the patient, resulting in cost savings at equal dose, or greater health benefits if HNHD is adopted.

Table 1. Financial and environmental values for three haemodialysis modalities and their cost-effectiveness

\begin{tabular}{|c|c|c|c|c|c|c|}
\hline \multirow{2}{*}{$\begin{array}{l}\text { Regime (per week) } \\
\text { Year }\end{array}$} & \multicolumn{2}{|c|}{$\begin{array}{c}\text { ICHD } \\
3 \times 4 \text { hours }\end{array}$} & \multicolumn{2}{|c|}{$\begin{array}{c}\text { HHD } \\
3 \times 4 \text { hours }\end{array}$} & \multicolumn{2}{|c|}{$\begin{array}{c}\text { HNHD } \\
6 \times 7 \text { hours }\end{array}$} \\
\hline & Year 1 & Year 2 & Year 1 & Year 2 & Year 1 & Year 2 \\
\hline Carbon footprint (ton $\mathrm{CO}_{2} \mathrm{eq}$, in 2010) ${ }^{\mathrm{a}}$ & $4.77^{b}$ & 3.82 & $4.14^{\mathrm{b}}$ & 3.31 & $9.00^{b}$ & 7.20 \\
\hline Cost of carbon $(£, \text { in } 2010)^{c}$ & 248.17 & 198.54 & 215.02 & 172.02 & 467.81 & 374.24 \\
\hline Cost of treatment $(£, 2010)^{d}$ & $28,131.88$ & $27,921.08$ & $27,921.08$ & $23,412.44$ & $35,896.76$ & $30,654.04$ \\
\hline Quality-adjusted life years (QALY) ${ }^{e}$ & 0.84 & 1.23 & 1.08 & 1.64 & 1.41 & 1.42 \\
\hline Total cost $(£ \text {, in } 2010)^{f}$ & $28,380.05$ & $28,119.62$ & $28,136.10$ & $23,584.46$ & $36,364.57$ & $31,028.28$ \\
\hline Cost per QALY (£, in 2010) & $33,490.33$ & $22,861.48$ & $25,852.85$ & $14,380.77$ & $25,458.70$ & $21,850.90$ \\
\hline ICER $^{g}$ & - & & $-£ 1,016.46$ & $-£ 11,061.37$ & $£ 14,007.92$ & $£ 15,308.78$ \\
\hline \multicolumn{7}{|c|}{ 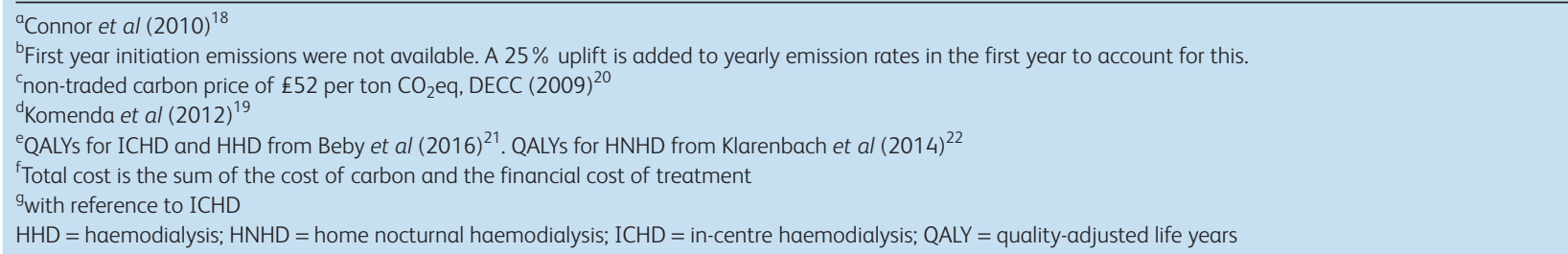 } \\
\hline
\end{tabular}


Our analysis only accounts for carbon dioxide equivalent emissions, which is one aspect of the problem, and does not represent the overall environmental damage of a treatment. As reviewed by Eckelman and Sherman, ${ }^{25}$ in the case of the USA, the healthcare sector is responsible for a large spectrum of emissions relative to total air pollution emissions, including acid rain (2\%), smog formation (10\%) among others. ${ }^{25}$ Accounting for these pollutants will increase the environmental footprint of the service. In our example of $\mathrm{HD}$, the difference between the financial costs of ICHD and HHD were large enough to not be affected by their carbon costs, but it is not possible without more information to say in which direction our results would be affected once we account for all possible environmental damages. Yet, a comprehensive monetary equivalent estimate of the damage does not exist.

Including environmental costs in a health economic evaluation will have further implications. First, the usual NICE acceptability threshold for an increase of one QALY is $£ 30,000$ and has to be reviewed upwards. Second, the decision maker does not directly incur the environmental costs immediately. In order to be effectively taken into account in the decision process, these costs should be incurred through some mechanism, for example by imposing a tax. This is based on the argument that any externality should be internalised at the source of the emission. Translating this simple logic in practice is difficult to implement.

\section{Conclusion}

Currently, the SDU assesses the environmental sustainability of the healthcare sector by focusing primarily on GHG emissions. This article has illustrated how GHG emissions can be evaluated and accounted for in an overall assessment. This is straightforward as there is research available on the monetary cost of carbon, allowing for its inclusion in an economic approach. Yet, economic activities, such as the delivery of healthcare, have a much broader impact on the environment as their emissions generate other pollution externalities. Unfortunately, there is no conversion value that allows for the internalisation of this externality and as a result these impacts continue to be ignored. A thorough sustainable approach to healthcare will have to find a way to account for the pollution attributed to its activities.

Long-term financial sustainability will be supported by an environmentally sustainable approach, and there are other co-benefits to be expected from it. ${ }^{26}$ Health co-benefits will come from a reduction of environmental pollutants harmful to health, but also by a change in behaviour typically conducive to a more active lifestyle with lower emissions. Quality of healthcare co-benefits have also been identified where less duplication, redundancy and waste will be supported by more environmentally driven objectives. $^{8}$

We illustrate a simple way to account for carbon emissions, but beyond the environmental price of environmental damages, achieving sustainability of the healthcare system will require more fundamental transformations - with a holistic integration of the sustainability objectives into the overall healthcare assessment. It does not seem to be a lack of interest in sustainable development by healthcare leaders, but a lack of means and metrics available to support sustainability as an important component in the delivery of health-related services that is currently the main constraint. ${ }^{27}$ Such goals can only be achieved with both the leadership from regulators and stakeholders, and engagement of the population to support changes toward a more environmentally sustainable provision of healthcare. ${ }^{10}$ However, a literature review of the evidence on environmental sustainably in the public healthcare in England concludes that the current policy framework is perceived to create barriers that do not support systematic changes. Improving environmental sustainability should be seen as an opportunity to review the efficiency of the healthcare system and implement cost saving as well as environmentally friendly measures that will support the long-term sustainability of the $\mathrm{NHS}^{8,28}$

\section{References}

1 Naylor C, Appleby J. Environmentally sustainable health and social care: Scoping review and implications for the English NHS. J Health Serv Res Policy 2013;18:114-21.

2 Financial sustainability of the NHS. National Audit Office, 2016.

3 National Institue for Excellence Health and Care Excellence. Developing NICE guidelines: the manual. London: NICE, 2014.

4 NHS Sustainable Development Unit. NHS England Carbon Emissions: Carbon Footprinting Report 2008 SDU, 2009.

5 NHS Sustainable Development Unit. Carbon Footprint update for NHS in England 2015. SDU, 2016.

6 Landrigan PJ, Fuller R, Acosta $\mathrm{N}$ et al. The Lancet Commission on pollution and health. The Lancet 2017.

7 The Department of Health and Social Care. The NHS Constitution for England. London: DH, 2015.

8 Naylor C, Appleby J. Sustainable health and social care: Connecting environmental and financial performance. King's Fund, 2012.

9 McGain F, Naylor C. Environmental sustainability in hospitals - a systematic review and research agenda. J Health Serv Res Policy 2014:19:245-52.

10 Ryan-Fogarty Y, O'Regan B, Moles R. Greening healthcare: Systematic implementation of environmental programmes in a university teaching hospital. J Clean Prod 2016;126:248-59.

11 Patrick R, Capetola T, Townsend M, Hanna L. Incorporating sustainability into community-based healthcare practice. Ecohealth 2011:8:277-89.

12 Desmond S. Implementing climate change mitigation in health services: the importance of context. J Health Serv Res Policy 2016;21:257-62.

13 Sustainable Development Unit. Care Pathways: Guidance on Appraising Sustainability - Emergency Department Visit. SDU, 2015.

14 NHS Sustainable Development Unit. Module: An Integrated Approach to Metrics. SDU, 2015.

15 Nichols A, Maynard V, Goodman B, Richardson J. Health, climate change and sustainability: A systematic review and thematic analysis of the literature. Environ Health Insights 2009;33:63-88.

16 Baboolal K, McEwan P, Sondhi S et al. The cost of renal dialysis in a UK setting - A multicentre study. Nephrol Dial Transplant 2008:23:1982-9.

17 National Institute for Clinical Excellence. Guidance on home compared with hospital haemodialysis for patients with end-stage renal failure. London: NICE, 2002.

18 Connor A, Lillywhite R, Cooke MW. The carbon footprints of home and in-center maintenance hemodialysis in the United Kingdom. Hemodial Int 2011:15:39-51.

19 Komenda P, Gavaghan MB, Garfield SS, Poret AW, Sood M. An economic assessment model for in-center, conventional home, and more frequent home hemodialysis. Kidney Int 2012;81:307-13.

20 Department of Energy and Climate Change. Carbon valuation in UK policy appraisal: a revised approach. DECC, 2009:1-128.

21 Beby AT, Cornelis T, Zinck R, Liu FX. Cost-effectiveness of high dose hemodialysis in comparison to conventional in-center hemodialysis in the Netherlands. Adv Ther 2016;33:2032-48. 
22 Klarenbach SW, Tonelli M, Chui B, Manns B]. Economic evaluation of dialysis therapies. Nat Rev Nephrol 2014;10:644-52.

23 National Institute for Health and Care Excellence. How NICE measures value for money in relation to public health interventions. LGB10. NICE, 2013.

24 UK Renal Registry. 19th Annual Report of the Renal Association. NEPHRON 2017;1(Suppl 1):137.

25 Eckelman MJ, Sherman J. Environmental impacts of the U.S health care system and effects on public health. PLoS One 2016;11:1-14

26 Connor A, O'Donoghue D. Sustainability: The seventh dimension of quality in health care. Hemodialysis International 2012;16:2-5.
27 Ling T, Pedersen J, Drabble S et al. Sustainable Development in the National Health Service (NHS). Rand Europe, 2012.

28 Pencheon D. Making health care more sustainable: The case of the English NHS. Public Health 2015;129:1335-43.

Address for correspondence: Dr Laure de Preux, Imperial College Business School, Imperial College London, South Kensington Campus, London SW7 2AZ, UK. Email: L.dePreux@imperial.ac.uk

\section{NGC natana

\section{New to systematic reviewing, or need} to improve your critical appraisal skills?

Our intensive 1-day courses will introduce you to the key principles of critical appraisal and systematic reviewing, and equip you with the basic skills to put your knowledge into practice.

> Introduction to critical appraisal

> Systematic reviews and meta-analysis in action

Discount available if both courses are booked together

\section{'Informative and practical'}

Dr Rajeswari Ramaraj

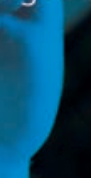

CPD

credits

For course dates and more information visit: www.ngc.ac.uk/training-courses 